\title{
Tetralogía de Fallot: diagnóstico, cirugía correctiva y manejo postoperatorio en Unidad de Cuidados Intensivos Cardiológicos Pediátricos, presentación de caso clínico y revisión bibliográfica.

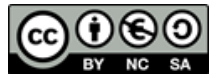

Tetralogy of Fallot: diagnosis, corrective surgery and postoperative management in Pediatric Cardiological Intensive Care Unit, presentation of a clinical case and literature review.

Dra. Ana Cristina Moncayo Torres. ${ }^{1} \&$ Dra. Mercedes Hernández Duarte ${ }^{2}$

Recibido: 17-10-2018 / Revisado: 20-11-2018 /Aceptado: 05-12-2018/ Publicado: 05-01-2019

\begin{abstract}
.
DOI: https://doi.org/10.33262/cienciadigital.v3i1.249

Tetralogy of Fallot is a congenital heart disease that is characterized by infundibular stenosis of the pulmonary artery, aortic thrust, ventricular septal defect, and hypertrophy of the right ventricle. It is the most prevalent cyanogenic congenital heart disease, representing between $3.5 \%$ and $8 \%$ of congenital heart defects. We present the clinical case of a minor infant patient, with a prenatal diagnosis of an interventricular defect that at its birth presented systolic murmur and was diagnosed with Tetralogy of Fallot after echocardiographic assessment, initially receiving pharmacological treatment and to whom, at 10 months of age a surgical procedure was performed to correct Tetralogy of Fallot with infundibular patch, ligature of Persistent Arteriosus Ductus, closure of Interventricular Communication and closure of Ostium Secundum Interauricular Communication, receiving later management in the Cardiological Intensive Care Unit of Children's Hospital "Dr. Roberto Gilbert Elizalde "of the city of Guayaquil in the month of September of the year 2018.
\end{abstract}

Keywords: Tetralogy of Fallot, Interventricular Communication, Interauricular Communication, congenital cardiopathies, murmurs.

\footnotetext{
${ }^{1}$ Hospital de Niños “Dr. Roberto Gilbert Elizalde”, Guayaquil, Ecuador, anacris2601@ hotmail.com

${ }^{2}$ Hospital de Niños "Dr. Roberto Gilbert Elizalde", Guayaquil, Ecuador, mhernandezduarte@ gmail.com
} 


\section{Resumen.}

La Tetralogía de Fallot es una cardiopatía congénita que se caracteriza por presentar estenosis infundibular de la arteria pulmonar, cabalgamiento aórtico, comunicación interventricular e hipertrofia del ventrículo derecho. Es la cardiopatía congénita cianógena más prevalente, representando entre el 3.5 a $8 \%$ de los defectos cardiacos congénitos. Se presenta a continuación el caso clínico de un paciente lactante menor, con diagnóstico prenatal de defecto interventricular que a su nacimiento presentó soplo sistólico y recibió diagnóstico de Tetralogía de Fallot tras valoración ecocardiográfica, recibiendo inicialmente tratamiento farmacológico y a quién, a los 10 meses de edad se le realizó procedimiento quirúrgico para corrección de Tetralogía de Fallot con parche infundibular, ligadura de Ductus Arterioso Persistente, cierre de Comunicación Interventricular y cierre de Comunicación Interauricular Ostium Secundum, recibiendo posteriormente manejo en la Unidad de Cuidados Intensivos Cardiológicos del Hospital de Niños "Dr. Roberto Gilbert Elizalde” de la ciudad de Guayaquil en el mes de septiembre del año 2018.

Palabras claves: Tetralogía de Fallot, Comunicación Interventricular, Comunicación Interauricular, cardiopatías congénitas, soplos.

\section{Introducción.}

La tetralogía de Fallot representa entre 3.5 y $8 \%$ de los defectos cardiacos congénitos y afecta aproximadamente a 1 de cada 2,400 nacidos vivos. Se trata de un complejo de alteraciones en la arquitectura cardiaca que consta de 4 componentes: aorta cabalgada, estenosis pulmonar, comunicación interventricular e hipertrofia de ventrículo derecho. Entre sus manifestaciones clínicas se presenta retraso en el desarrollo tanto físico y psicomotor, además de manifestaciones de hipoxia crónica, como la acropaquia. El estado de hipoxia crónica condiciona el desarrollo de policitemia, aumentando así, además, la viscosidad sanguínea y aumentando el riesgo de desarrollo de trombosis en diversos órganos, por lo que es importante un diagnóstico precoz y manejo adecuado con corrección definitiva, para así evitar potenciales complicaciones.

\section{Metodología}

Presentación de caso clínico de un paciente con diagnóstico prenatal de defecto interventricular, presentando, posterior a su nacimiento, diagnóstico ecocardiográfico de Tetralogía de Fallot, en quien se realizó cirugía correctiva y manejo postquirúrgico en el área de Cuidados Intensivos Cardiológicos Pediátricos.

\section{Caso Clínico}


Paciente de sexo masculino con diagnóstico prenatal de alteración ventricular, quien a su nacimiento presentó soplo sistólico y tras valoración ecocardiográfica se diagnosticó Tetralogía de Fallot. Se manejó desde entonces con Furosemida 4 mg vía oral cada 8 horas, Captopril 4 mg vía oral cada 8 horas y Espironolactona 9 mg vía oral cada día.

A los 4 meses de edad acuden a control por consulta externa de Cardiología del Hospital de Niños Dr. Roberto Gilbert Elizalde, en donde presenta al examen físico precordio activo derecho, ruidos cardiacos rítmicos, soplo sistólico 4/6 en meso y 2R hiperfonético, saturación de $\mathrm{O} 2$ de $99 \%$ y frecuencia cardiaca de $1331 \mathrm{pm}$.

Las pruebas de laboratorio presentan hemoglobina de 11.2 con hematocrito de 34.1 . El electrocardiograma muestra trastornos inespecíficos de la repolarización con $\mathrm{S}$ profunda en V3 y R alta en V4 e hipertrofia septal. En la radiografía de tórax se observa levantamiento del ápex a expensas de crecimiento de cavidades derechas y disminución del calibre de la arteria pulmonar, dando una imagen de "corazón en forma de bota" Gráfico 1.

Gráfico 1 Radiografía de tórax pre quirúrgica que muestra silueta cardiaca en forma de "bota" o "sueco".

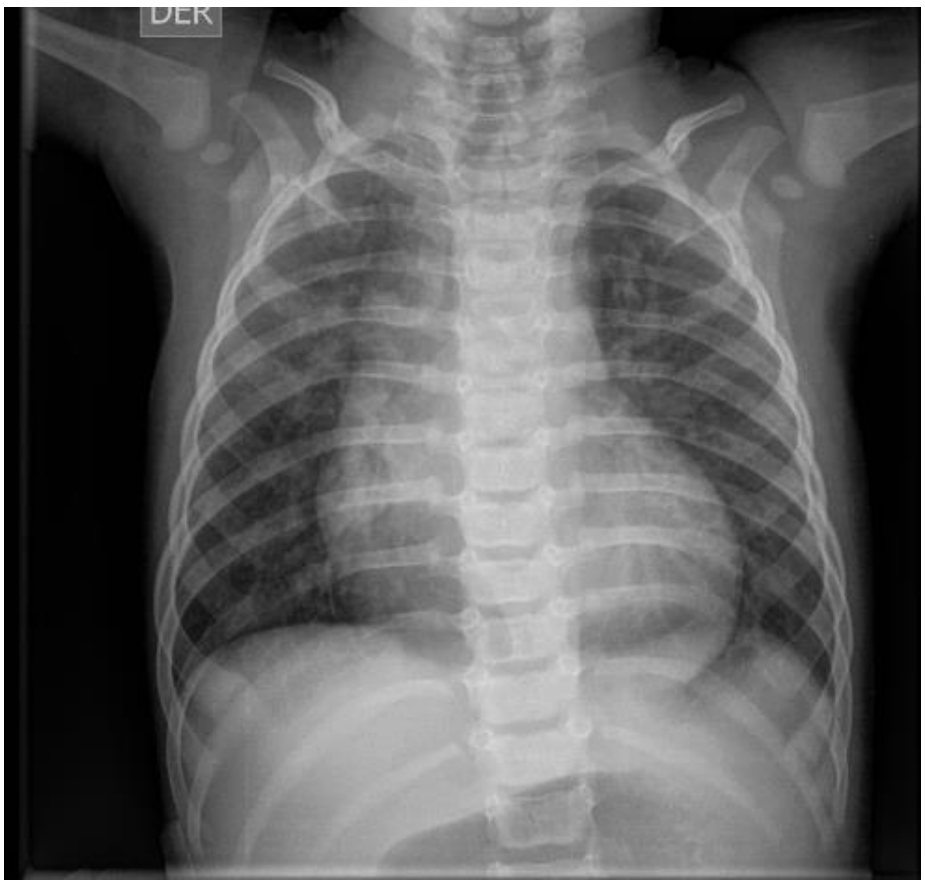

Elaborado por: Grupo de investigación.

Se realizó ecocardiograma, en el que se observan cavidades izquierdas dilatadas: DDVI $3.9 \mathrm{~cm}$ (zscore 5.1), DSVI $2.3 \mathrm{~cm}$ (zscore 3.6), foramen oval permeable mínimo con shunt de izquierda a derecha, comunicación interventricular subaórtica grande de $1.4 \mathrm{~cm}$, aorta que cabalga el SIV, válvula aórtica tricúspide con leve prolapso de velo e insuficiencia aórtica leve, tracto de salida del ventrículo izquierdo libre, flujo laminar, tracto de salida del 
ventrículo dercho con flujo turbulento desde la región infundíbulo-valvular infundíbulo proximal $29 \mathrm{mmHg}$ infundíbulo distal $22 \mathrm{mmHg}$ valvular gte máximo $37 \mathrm{mmHg}$ medio $20.5 \mathrm{mmHg}$ Anillo Pulmonar $1.3 \mathrm{~cm}$ ( z score 1.8) Tronco de AP ( z score 2.4) $1.5 \mathrm{~cm}$ RPD $0.8 \mathrm{~cm}$ ( z score 1.5 ) RPI $0.9 \mathrm{~cm}$ ( z score 2.8). Gráfico 2.

Gráfico 2 Ecocardiograma prequirúrgico

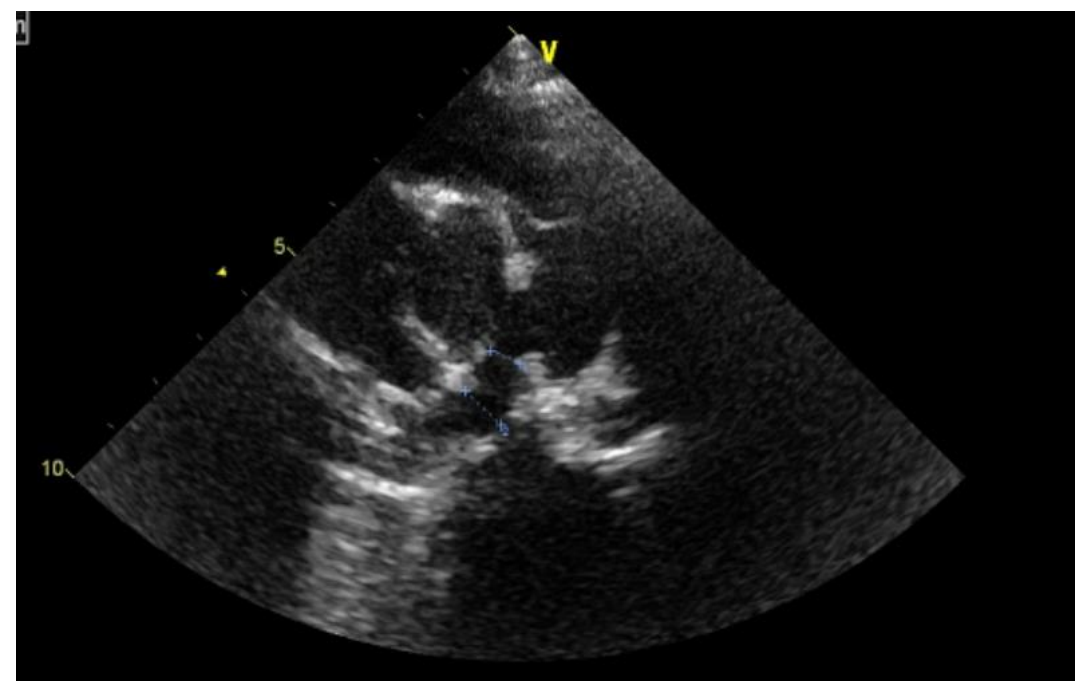

Elaborado por: Grupo de investigación.

Con estos hallazgos, se realizó programación para cirugía correctiva.

A los 10 meses de edad se realizó procedimiento quirúrgico: corrección de Tetrallogia de Fallot con parche infundibular + ligadura de ductus + cierre de CIV + cierre de CIA OS. Se realizó esternotomia media, timectomía total, se abre el pericardio, se administra heparina se realiza canulación aórtica y bicava con sus respectivos torniquetes, se entra en circulación extracorpórea, se enfría a 32 grados, se coloca aguja de plegia y vent AV en vena pulmonar superior derecha, se clampea la aorta, se administra cardioplegia lograndose paro electromecanico, se abre el infundibulo pulmonar, se reseca hipertrofia muscular, se cierra el defecto interventricular sub aortico con membrana de gorotex, se pasa dilatador Jegard 11 $\mathrm{mm}$ a nivel de la valvula pulmonar el cual pasa sin inconveniente (valor Z-0 de la pulmonar= 10.7), se cierra la infundibulotomia con parche de pericardia fijado en glutaraldheido por 15 min, se abre la auricula derecha, se cierra foramen oval permeable con sutura directa, se cierra la auricula, se realiza maniobras de extraccion de aire, se declampea la aorta el corazón reasume su actividad con ritmo sinusal, se calienta el paciente y se sale de circulacion extracorporea, se realiza ultrafiltracion modificada, se administra protamina y se decanula, se chequea hemostasia se coloca surgicel, cables de marcapaso transitorios ventriculares y drenaje de silicon mediastinico pleural derecho, se mide presiones del ventriculo derecho derecho la maxima de $27 \mathrm{mmhg}$ y de arteria pulmonar de $26 \mathrm{mmhg}$, se cierra el esternon con puntos de PDX -0 y el torax con tecnica habitual. 
CEC: 93 min

ClAo: $80 \mathrm{~min}$

Posterior a la cirugía, se lo recibe en el área de UCI Cardio bajo efectos de sedación, intubado con tubo 4 con balón fijado en 11, con protector gástrico, en lo hemodinámico llega al área hipotenso, febril $38.4^{\circ} \mathrm{c}$, taquicárdico(FC: $188 \mathrm{lpm}$ ), por lo que se decide carga de plasma a $10 \mathrm{cc} / \mathrm{kg}$ por 2 ocasiones y se pauta esquema antibiótico profiláctico con cefalosporina intravenosa de primera generación.

Se indica mantenerlo en Ventilacion mecánica, modo SIMV Fr 16 - P insp 18 - T insp 0.75 PSV 10 - PEEP 5 - Disp flujo 1.0 - Fi o2: 50. Se inicia infusión continua de heparina, milrinona y epinefrina.

Se lo extuba a las 5 horas, sin complicaciones.

Se realiza ecocardiograma que evidencia CIV cerrada, gradiente infundibular $11 \mathrm{mmHg}$, IT 45 - 50 mmhg, ventrículo derecho hipertrófico, ventrículo izquierdo con adecuada función, no derrame pericárdico por lo que se mantuvo con apoyo de milrinona $0,6 \mathrm{mcg} / \mathrm{kg} \mathrm{min}$ y epinefrina a $0.05 \mathrm{mcg} / \mathrm{kg} / \mathrm{min}$ y se inicia diurético a dosis respuesta para un balance neutro. Debido a ventrículo derecho restrictivo y mala perfusión distal y balance negativo se administró albúmina al 5\%.

A las 48 horas de postquirúrgico por signos vitales dentro del percentil 50 para la edad y por estabilidad hemodinámica, se decide suspender epinefrina e iniciar captopril y milrinona.

Se mantuvo con ketorolaco e ibuprofeno para analgesia, con adecuada tolerancia.

A los 5 días de postquirúrgico se realiza ecocardiograma de control en el que se observa septum interventricular sin corto circuito residual, tracto salida ventrículo derecho sin obstrucción, insuficiencia aortica leve, hipertrofia ventrículo derecho, pero con buena función. Gráfico 3.

Gráfico 3. Radiografía de tórax postquriúrgica 


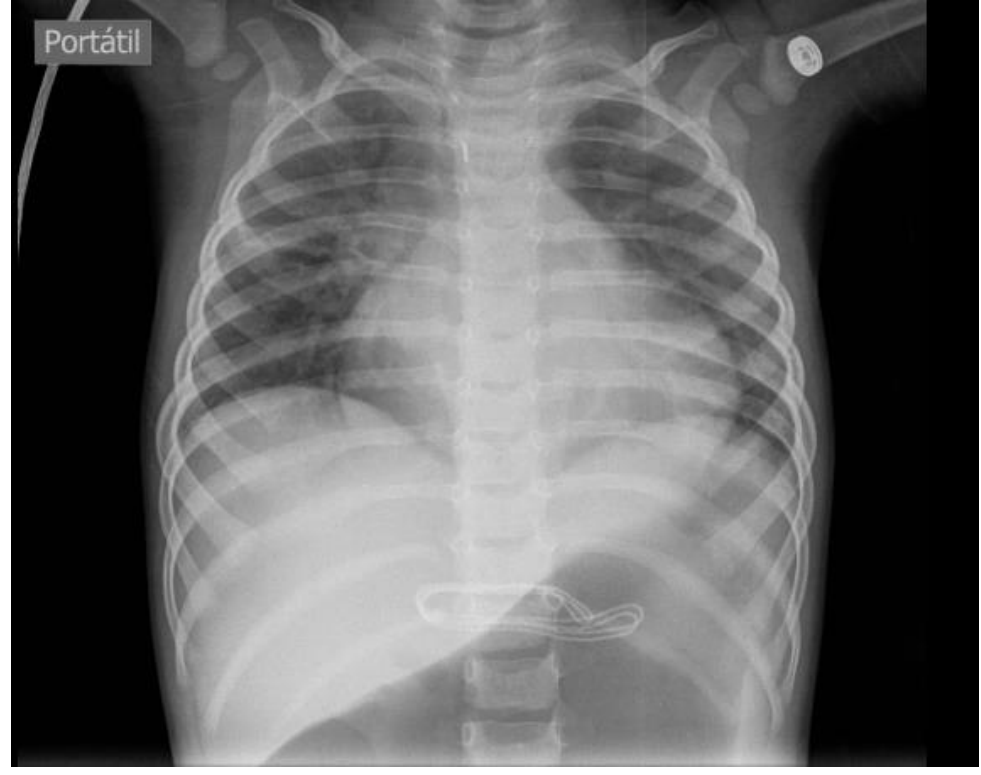

Elaborado por: Grupo de investigación.

Se observa radiografía de tórax con buen estado de parénquima pulmonar. Gráfico 4.

Gráfico 4. Ecocardiograma postquirúrgico

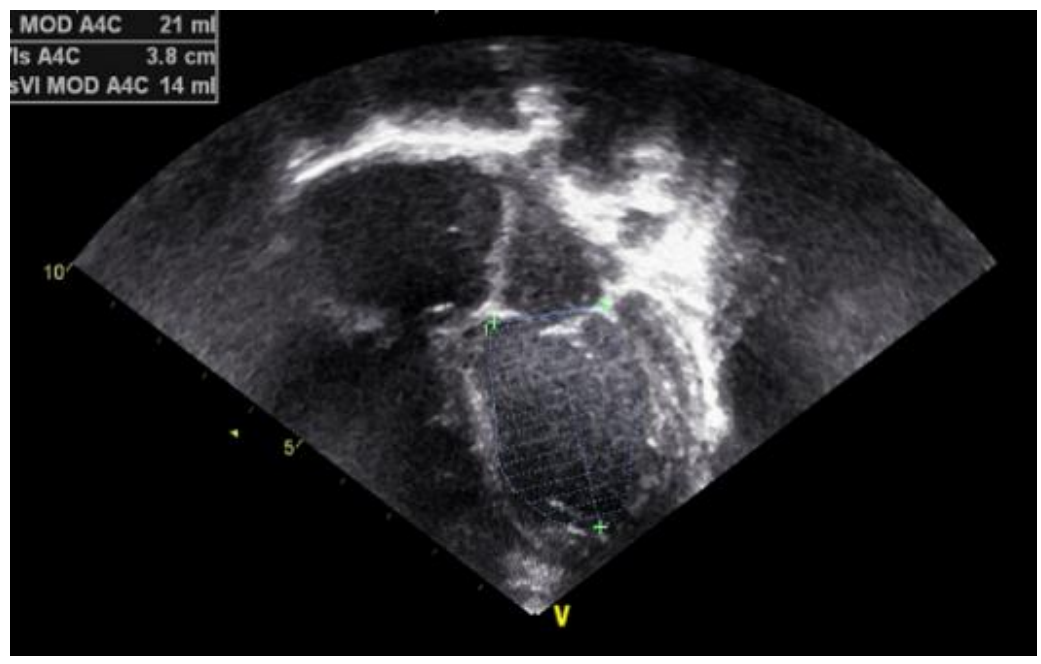

Elaborado por: Grupo de investigación.

Exámenes de laboratorio de control dentro de parámetros adecuados, por lo que se decide alta hospitalaria, indicando manejo con ibuprofeno, furosemida, espironolactona, captropril.

A los 7 días, se realiza control por consulta externa de Cardiología, presentado al examen físico, ruidos cardiacos rítmicos, soplo sistólico $2 / 6$ en meso y se realiza rastreo ecocardiográfico observando CIV residual pequeña de $3 \mathrm{~mm}$, IT leve de baja velocidad, no derrame pericárdico ni pleural y se indica mantener igual medicación. 


\section{Resultados y Discusión}

La tetralogía de Fallot (TOF) incluye las siguientes características principales:

- Estenosis de la arteria pulmonar.

- Comunicación intraventricular

- Desviación del origen de la aorta hacia la derecha.

- Hipertrofia ventricular derecha concéntrica.

EPIDEMIOLOGÍA: la prevalencia de Tetralogía de Fallot en los Estados Unidos es de aproximadamente 4 a 5 por 10,000

Nacimientos vivos. Este defecto representa aproximadamente del 7 al 10 por ciento de los casos de cardiopatías cardiacas congénitas y es una de las lesiones cardíacas congénitas más comunes que requieren intervención en el primer año de vida. Ocurre de igual manera en hombres y mujeres.

ANATOMÍA: se desconoce la anomalía embriológica exacta que explica la Tetralogía de Fallot, pero se reconoce que, durante el desarrollo, existe una desviación anterior y cefálica del infundibular, lo cual resulta en un defecto septal ventricular mal alineado (VSD), con la sobrevaloración de la raíz aórtica lo que conduce a una obstrucción del flujo de salida del ventrículo derecho posterior.

Se cree que la hipertrofia del ventrículo derecho es una respuesta al defecto septal ventricular mal alineado grande y al ventrículo derecho, lo que da como resultado una obstrucción del flujo de salida con presión sistólica ventricular derecha resultante.

Defecto del tabique ventricular: el septo ventricular mal alineado en la Tetralogía de Fallot, es más comúnmente un defecto subaórtico grande mal alineado localizado hacia la región perimembranosa del septo.

El VSD puede extenderse hacia el tabique muscular. Raramente hay otros defectos del tabique ventricular muscular.

Obstrucción del flujo de salida del ventrículo derecho: la obstrucción del flujo de salida del ventrículo derecho a menudo se encuentra en niveles múltiples:

- La desviación anterior y cefálica del tabique infundibular da como resultado una obstrucción subvalvar.

- La hipertrofia de las bandas musculares en esta región puede acentuar aún más la obstrucción subvalvar.

- El anillo de la válvula pulmonar suele ser hipoplásico, aunque en algunos casos es de tamaño normal.

- La válvula pulmonar en sí es frecuentemente bicúspide y estenótica. 
Aorta superior: la aorta superior es una anomalía congénita en la que la aorta se desplaza hacia la derechoa, justo sobre el VSD en lugar del ventrículo izquierdo. Esto resulta en el flujo de sangre de ambos ventrículos hacia la aorta.

Anomalías cardíacas asociadas: hay una serie de características anatómicas asociadas con frecuencia, que son importantes buscar cuando se evalúa a un paciente con Tetralogía de Fallot, ya que afectan la terapéutica a utilizarse.

Las anomalías cardíacas asociadas ocurren en aproximadamente el 40 por ciento de los pacientes con Tetralogía de Fallot.

Factores genéticos: aunque la Tetralogía de Fallot puede presentarse como parte de un síndrome conocido, esta lesión típicamente ocurre esporádicamente sin otras anomalías. En pacientes con Tetralogía de Fallot no relacionados con alteraciones sindrómicas, se han informado las siguientes anomalías genéticas:

En un estudio de 114 pacientes con Tetralogía de Fallot, el 4 por ciento de los pacientes tenía mutaciones en el factor de transcripción NKX2.5., que parece tener un papel en el desarrollo cardíaco.

En estudios de genoma de pacientes con Tetralogía de Fallot no sindrómico y sus padres, se estimó que las variantes numéricas estaban presentes en aproximadamente el 10 por ciento de los casos esporádicos de Tetralogía de Fallot, comparado con menos del 0.1 por ciento en controles en varias ubicaciones cromosómicas.

Varios informes han asociado Tetralogía de Fallot con mutaciones en TBX1 y ZFPM2.

El polimorfismo MTHFR también se ha asociado con un mayor riesgo de desarrollo de Tetralogía de Fallot.

Se requiere investigación adicional para determinar el papel de estas mutaciones y polimorfismos en la evolución de la Tetralogía de Fallot.

Aproximadamente el 15 por ciento de los pacientes con Tetralogía de Fallot presentan síndromes asociados:

Síndrome de Down (trisomía 21), Síndrome de Alagille (mutaciones en JAG1), Síndrome de Di George y síndromes velocardiofaciales (supresión en el cromosoma 22q11). Puede haber genes de susceptibilidad para Tetralogía de Fallot dentro de la última región del cromosoma 22q11 en niños sin anomalías extracardíacas, y el síndrome de deleción 22q11.2 no se reconoce en muchos pacientes adultos con Tetralogía de Fallot.

Patología: las consecuencias fisiológicas de la TOF dependen en gran medida del grado de obstrucción del flujo de salida del ventrículo derecho. Dado que el VSD es típicamente 
grande y no restrictivo, la presión en el ventrículo derecho refleja la del ventrículo izquierdo. Como resultado, la dirección del flujo de sangre a través del VSD se determinará por la ruta de menor resistencia para el flujo sanguíneo, no por el tamaño del VSD. Si la resistencia al flujo sanguíneo a través del tracto de salida del ventrículo derecho obstruido es menor que la resistencia a fluir de la aorta a la circulación sistémica, la sangre se desviará naturalmente del ventrículo izquierdo al ventrículo derecho y al lecho pulmonar. En esta situación, hay predominantemente una derivación de izquierda a derecha y el paciente no presentará cianosis.

A medida que aumenta el grado de obstrucción del flujo de salida del ventrículo derecho, la resistencia al flujo sanguíneo hacia el lecho pulmonar también aumenta. Si la obstrucción ventricular derecha es lo suficientemente significativa como para aumentar la resistencia, será más fácil para la sangre cruzar el VSD desde el ventrículo derecho al ventrículo izquierdo y salir por la aorta, que ahora se convierte en el camino de menor resistencia. Esta derivación de derecha a izquierda a través del VSD da como resultado un gran volumen de sangre desaturada que ingresa a la circulación sistémica, lo que provoca que el paciente presente cianosis.

Una de las características fisiológicas de la Tetralogía de Fallot, es que la obstrucción del flujo de salida del ventrículo derecho puede fluctuar, provocando así, que un individuo con cianosis mínima pueda desarrollar un aumento dinámico en el ventrículo derecho y obstrucción del tracto de salida con un aumento posterior de la derivación de derecha a izquierda, provocando el desarrollo de cianosis. En la situación más dramática, puede haber cerca de oclusión del flujo de salida del ventrículo derecho, un tracto con cianosis profunda provocando episodios de hipercianosis; la etiología exacta de estos episodios no está clara, aunque ha habido una serie de mecanismos propuestos, incluyendo contractilidad infundibular incrementada, vasodilatación periférica, la hiperventilación y estimulación de los mecanorreceptores del ventrículo derecho.

\section{Características clínicas:}

Presentación: la presentación clínica del paciente con Tetralogía de Fallot depende del grado de obstrucción del flujo de salida del ventrículo derecho:

- Los niños con obstrucción severa y flujo pulmonar inadecuado típicamente se presentan en el periodo neonatal inmediato con cianosis profunda.

- Se pueden observar niños con obstrucción moderada y flujo pulmonar y sistémico equilibrado en niños en quienes se está realizando evaluación por auscultarse un soplo durante el examen físico; estos niños también pueden presentar episodios de hipercianosis, los cuales se presentan durante los períodos de agitación.

- Los niños con obstrucción mínima pueden presentar sobrecirculación pulmonar e insuficiencia cardíaca. 
Además, algunos recién nacidos afectados serán detectados por una evaluación en la que se presenta alteración en la oximetría de pulso durante el examen físico.

La mayoría de los niños con Tetralogía de Fallot son sintomáticos y cianóticos; existe un subgrupo, sin embargo, con típicas morfología y hemodinámica que permanece clínicamente asintomáticos durante un período de tiempo (variante rosa). En general, cuanto más temprana sea la aparición de la hipoxemia sistémica, más probable es que exista estenosis del tracto de salida pulmonar o atresia.

Examen físico: en la inspección, las personas con Tetralogía de Fallot, suelen estar cómodas y sin distrés respiratorio. Sin embargo, durante los episodios hipercianóticos (tet), se volverán hiperpneicos, y los bebés a menudo se agitan. Si la cianosis está presente, se ve más fácilmente en los lechos de las uñas y los labios. En la palpación, se puede apreciar un impulso ventricular derecho prominente y, en ocasiones, presentan soplo sistólico. La hepatomegalia es infrecuente. Los pulsos periféricos suelen ser normales, aunque la presencia de pulsos prominentes puede sugerir la existencia de un ductus arterioso permeable o colaterales aorticopulmonares. Auscultación cardíaca: en la auscultación, el primer ruido cardíaco es normal y el segundo ruido acenetuado porque el componente pulmonar rara vez es audible. El tercero y cuarto ruidos cardiacos son infrecuentes. Se puede escuchar un clic sistólico temprano a lo largo del borde esternal izquierdo, que se cree que se debe a que fluye hacia la aorta ascendente dilatada.

Soplo: el soplo en la Tetralogía de Fallot se debe principalmente a la obstrucción del flujo de salida del ventrículo derecho y no a la VSD. El soplo es típicamente crescendo-decrescendo con una calidad de eyección sistólica áspera, el cual se aprecia mejor a lo largo del borde esternal medio izquierdo y superior con irradiación posterior. Puede, sin embargo, tiene una calidad más regurgitante que puede confundirse fácilmente con un VSD. El soplo se debe tanto al grado de obstrucción como a la cantidad de flujo a través de la obstrucción. En Tetralogía de Fallot, a diferencia de la estenosis pulmonar valvular aislada, la cantidad de flujo a través de la derecha el tracto de salida ventricular disminuirá a medida que la obstrucción aumenta, debido a la derivación de la sangre de derecha a izquierda a través del VSD. Así, a medida que aumenta la obstrucción, el soplo se hará más suave. Durante los episodios hipercianóticos severos, el soplo puede desaparecer debido al flujo marcadamente disminuido a través de la obstrucción.

Diagnóstico: el diagnóstico de la Tetralogía de Fallot generalmente se realiza mediante ecocardiografía. Otras pruebas que son utilizadas a menudo durante la evaluación de la Tetralogía de Fallot incluyen electrocardiograma y radiografía de tórax.

Los hallazgos de estos estudios a menudo son sugerentes, pero no concluyentes para el diagnóstico de Tetralogía de Fallot.

El cateterismo cardíaco a veces es necesario para delinear la anatomía y hemodinámica. 
Diagnóstico prenatal: las mejoras en la detección prenatal y la ecocardiografía fetal han llevado a un aumento en el diagnóstico prenatal de la Tetralogía de Fallot. El diagnóstico fetal permite una planificación avanzada para parto y manejo perinatal, lo cual es particularmente importante si hay evidencia de severos defectos en la obstrucción del tracto de salida ventricular que requiere tratamiento con prostaglandinas para mantener la permeabilidad del conducto.

Ecocardiografía: la ecocardiografía bidimensional y el examen Doppler permiten la evaluación de todas las características esenciales de TOF y tiene un papel crucial en el diagnóstico y preoperatorio. La evaluación ecocardiográfica completa puede obviar la necesidad de otras imágenes o estudios diagnósticos antes de la reparación quirúrgica. La mayor parte de la información se puede lograr con ecocardiograma transtorácico, pero en ocasiones la ecocardiografía transesofágica.

Un estudio completo debe abordar:

-La ubicación y el número de VSDs.

-La anatomía y la gravedad de la obstrucción del tracto de salida del ventrículo derecho.

-La arteria coronaria y la anatomía del arco aórtico.

-La presencia de cualquier anomalía asociada.

Defecto del tabique ventricular: la VSD característica grande y mal alineada debe evaluarse en múltiples vistas. El grado de anulación aórtica se puede evaluar mejor en el eje largo paraesternal y en las vistas apicales (película 1 y película 2). La extensión del defecto del tabique membranoso, debajo de la válvula tricúspide, y en el tabique de salida del infracristal se ve bien en el paraesternal vista de eje corto; la extensión a la región supracristal, si está presente, se puede ver en esta vista también la extensión potencial del defecto posteriormente hacia el tabique de entrada, o apicalmente en el tabique trabecular, puede ser examinado en vistas apicales; el transductor es barrido a través de imágenes en serie de planos desde las vistas más caudales del tabique de entrada hasta las vistas anguladas anteriormente, mostrando la anulación de la aorta en continuidad con las válvulas mitral y tricúspide.

Las vistas subcostales también son útiles para delinear los límites de la VSD, lo que permite particularmente visualizar la representación de la relación entre el defecto y las válvulas tricúspide y aórtica. La vista oblicua derecha subcostal, obtenida al girar el transductor en sentido antihorario desde la vista coronal, también es útil para identificar cualquier posible extensión del defecto en la región supracristal.

En los casos raros de VSD restrictiva en esta lesión, esta vista también define la válvula tricúspide anormal. 
Obstrucción del flujo de salida del ventrículo derecho: los múltiples niveles y la gravedad de la obstrucción en el tracto de salida del ventrículo derecho también se puede evaluar mediante ecocardiografía.

El eje corto paraesternal y las vistas coronal y sagital subcostal permiten el mejor examen del infundíbulo y válvula pulmonar. Estas vistas demuestran la desviación anterior de la conal.

La talla del anillo pulmonar generalmente hipoplásico puede evaluarse y compararse con valores normales para el tamaño del paciente y superficie corporal. Esto es importante para establecer la necesidad potencial de un parche transanular. La válvula pulmonar puede aparecer engrosada y abovedada en estas vistas.

Arterias pulmonares: se valora el tamaño y anatomía de la arteria pulmonar principal, la pulmonar, la confluencia arterial, y las arterias pulmonares de rama proximal. Las arterias pulmonares de rama proximal se deben evaluar tan distalmente como sea posible, con vistas parasternas altas y en muescas supraesternales vistas en eje largo y corto, dirigiendo el transductor en el tórax izquierdo y derecho.

Arterias coronarias: la anatomía coronaria proximal debe definirse ecocardiográficamente en pacientes con Tetralogía de Fallot. Además de examinar la anatomía coronaria en la vista tradicional de eje corto, el examen debe incluir barrer el transductor anterior al tracto de salida pulmonar en las vistas de eje largo y corto paraesternales. Esto permite la identificación de variaciones en la anatomía coronaria, incluido el origen de la descendencia anterior izquierda de la arteria coronaria derecha o suministro de doble vaso a la distribución descendente anterior

Arco aórtico: el arco aórtico y los patrones de ramificación de las arterias braquiocefálicas son definidos en la muesca supraesternal en las vistas de eje largo y corto.

Septos auriculares y ventriculares: la evaluación ecocardiográfica de los pacientes con Tetralogía de Fallot se

completa utilizando imágenes bidimensionales y mapeo de flujo de color del tabique auricular y del septo ventricular en múltiples planos de imagen. Esta evaluación define defectos auriculares y defectos del tabique ventricular y evalúa las posibles anomalías del retorno venoso pulmonar y sistémico y la rara aparición asociada de lesiones obstructivas del lado izquierdo.

Evaluación ecocardiográfica hemodinámica: En pacientes con obstrucción mínima del tracto de salida del ventrículo derecho, el gradiente a través de la derecha el tracto de salida ventricular será bajo, la presión arterial pulmonar estimada será elevada y la derivación a través del VSD, según lo evaluado por Doppler pulsado y el mapeo de flujo de color, será 
predominantemente de izquierda a derecha durante gran parte del ciclo cardíaco. En pacientes con una derivación grande de izquierda a derecha, la dilatación de la aurícula izquierda y el ventrículo izquierdo puede ser evidente por Imágenes bidimensionales. En pacientes con obstrucción del tracto de salida del ventrículo derecho más grave, la presión arterial pulmonar estimada es normal, y Doppler pulsado y mapeo de flujo de color demuestran un aumento de la derivación de derecha a izquierda en el defecto del tabique ventricular durante el ciclo cardíaco.

Cateterización cardíaca: aunque la ecocardiografía puede revelar la anatomía en muchos pacientes con Tetralogía de Fallot, el cateterismo cardíaco puede ser necesario para delinear aún más la estructura. Es particularmente útil para evaluar los niveles de obstrucción del flujo de salida del ventrículo derecho, rama pulmonar, estenosis o hipoplasia arterial, anatomía de la arteria coronaria, presencia de colaterales aorticopulmonares y presencia de defectos septales ventriculares accesorios.

Los hallazgos hemodinámicos en el cateterismo típicamente revelan un llenado normal o levemente elevado de las presiones. Las presiones sistólicas ventriculares izquierda y derecha son iguales y sistémicas debido a la presencia de la gran VSD. Las presiones de la arteria pulmonar son normales o bajas. Las saturaciones indicarán el grado de derivación de derecha a izquierda.

La evaluación angiográfica debe estar orientada hacia la información que se necesita.

El cateterismo cardíaco también puede desempeñar un papel terapéutico en algunos pacientes con Tetralogía de Fallot. La valvuloplastia de la válvula pulmonar puede mejorar el flujo pulmonar en muchos niños; también puede provocar un aumento en el tamaño del anillo de la válvula pulmonar que puede disminuir la necesidad de parche transanular.

\section{Diagnósticos diferenciales:}

En un lactante sin signos de insuficiencia cardíaca, con cianosis que varía al esfuerzo, soplo sistólico de eyección, radiografía de tórax con silueta en sueco e hipoflujo pulmonar, y ECG con signos de sobrecarga de presión de cavidades derechas, debe sospecharse esta malformación. La transposición simple de los grandes vasos suele presentarse en la primera semana de vida, con el cierre del ductus; en la radiografía se observa cardiomegalia (forma ovoide típica) e hiperflujo en los campos pulmonares. La estenosis pulmonar grave es una cardiopatía acianótica, ya que no existe cortocircuito. La atresia pulmonar con Comunicación Interventricular (anatomía muy similar a la Tetralogía de Fallot, pero sin flujo anterógrado por arteria pulmonar) suele requerir prostaglandinas o, si mantiene flujo pulmonar por el ductus o colaterales aorto-pulmonares, pueden auscultarse soplos continuos en vez del soplo sistólico eyectivo rudo de la Tetralogía de Fallot. La anomalía total del retorno venoso pulmonar, sea obstructiva o no, presenta signos de insuficiencia cardíaca con hiperflujo pulmonar en la radiografía de tórax, entre otras características. 


\section{Tratamiento:}

Ante verdaderas crisis de cianosis es perentorio realizar la corrección quirúrgica o, si ésta no fuera factible, una anastomosis sistémico-pulmonar. La cirugía reparadora consiste en ampliar el tracto de salida del VD y cerrar la CIV con un parche. Actualmente la edad óptima para la intervención es entre los 6 y los 9 meses, dependiendo de la anatomía. Con los avances en cirugía cardiovascular neonatal, la edad y el peso ya no son limitantes, y en casos favorables puede intentarse una corrección precoz. La lesión residual, muchas veces inevitable, es la insuficiencia pulmonar secundaria a la ampliación de la válvula. Ella conduce a estos niños inexorablemente a reoperaciones en la segunda infancia o adolescencia.

El tratamiento básico de la tetralogía de Fallot es quirúrgico. Tras la realización del ecocardiograma que nos dará el diagnóstico exacto de la malformación y de las lesiones asociadas, el cateterismo cardiaco y la angiocardiografía darán el enfoque óptimo del tratamiento quirúrgico. Las lesiones asociadas son muy numerosas y su repercusión en la cirugía de la tetralogía es variable, según el grado de severidad de ellas:

- Leves (no aumentan el riesgo de corrección total): Foramen oval permeable, Conducto arterioso, Ventana aortopulmonar sin hipertensión pulmonar.

- Severas (aumento de riesgo de corrección total): Estenosis periféricas en el ostium de una o ambas ramas pulmonares, Arteria coronaria anómala que cruza el infudíbulo, Agenesia de la válvula pulmonar, Agenesia de una rama pulmonar.

- Muy severas (contraindican corrección total): Estenosis periféricas de las arterias pulmonares no abordables quirúrgicamente, Resistencias pulmonares elevadas, Comunicaciones interventriculares múltiples.

La actuación quirúrgica inmediata será en los casos con hipoxemia severa mantenida, acidosis grave y en los enfermos con crisis hipoxémicas repetidas. Con urgencia relativa se consideran los casos en los que la intervención quirúrgica es necesaria a corto plazo con el fin de evitar complicaciones que puedan ser graves como hipertensión pulmonar, poliglobulia y cianosis severa con incapacidad física importante y aquellos cuyo objetivo es la preparación anatómica adecuada para la corrección completa. Con urgencia electiva clínicamente toleran bien la cardiopatía

\section{Elección de la técnica:}

La indicación de cirugía "paliativa" o "correctora" se establecerá de acuerdo con la edad y las condiciones anatómicas de cada caso demostradas por angiocardiografía:

Técnica paliativa: Esta técnica aumenta el flujo pulmonary, asegura una cantidad suficiente de sangre oxigenada mediante la realización de Fístula Sistémico Pulmonar. Algunas de las circunstancias en que debe ser considerada la técnica paliativa son: 1. Niños con crisis hipoxéinicas repetidas. 2. Cuando la arteria coronaria atraviesa el tracto de salida del 
ventrículo derecho. 3. Cuando las arterias pulmonares son muy pequeñas. 4. Cuando hay lesiones cardiacas asociadas que repercuten en la reparación completa.

Existen diferentes tipos de fístulas sistémico-pulmonares, pero la que más se utiliza es la de Blalock- Taussig modifícada, por ser la que menos complicaciones tiene, ya que es más fácil de controlar el flujo que pasa a través de ella. Consiste en la conexión latero-lateral entre la arteria subclavia y la arteria pulmonar con un tubo de gore-tex de aproximadamente de $4 \mathrm{~mm}$ (el tamaño de la fistula va a limitar el flujo), con ello se aumenta el flujo en la arteria pulmonar independientemente de que pueda llegar sangre del corazón derecho. Se puede realizar en la parte derecha o izquierda. En la derecha es más fácil de hacer, y se asocia a menos casos de lesión del nervio frénico y laríngeo. Otros autores prefieren el acceso izquierdo para reservar el derecho para futuras intervenciones como la conexión cavo-pulmonar. No necesita C. E. C. Sin embargo, no puede ser una solución a muy largo plazo va que puede producirse hipertensión pulmonar y sobrecarga del ventrículo sistémico, así como deformar la anatomía de la arteria pulmonar, complicando o contraindicando la cirugía definitiva. Durante esta intervención suele ligarse el ductus. En algunos casos no se hace, y tras la cirugía se retira la perfusión de prostaglandinas. Si disminuye la Sat02, y se sospecha que el shunt tiene poco flujo, o que se ha obstruido, se reinicia la perfusión de PGE 1. hasta que se revise la fístula o se haga un nuevo B-T.

Otras fístulas sistémico-pulmonares: En el Blalock-Tausing clásico se secciona la arteria subclavia y se anastomosa terminolateralmente a una rama de la arteria pulmonar, El flujo al brazo afectado se realiza por colaterales, y entre las posibles complicaciones de esta cirugía está la isquemia de la extremidad del lado del shunt, que-aunque no es frecuente si lo es la disminución en el crecirmiento y el desarrollo del brazo afectado. Estas complicaciones se minimizan con el B-T modificado. - Potts, anastomosis entre la aorta descendente y la arteria pulmonar izquierda. - Waterston, anastomosis entre la aorta ascendente y la arteria pulmonar derecha.

Corrección completa: El fin de la reparación total es: Cierre del defecto ventricular. Apertura del tracto de salida del ventrículo derecho. Reparar la estenosis de la arteria pulmonar. La técnica quirúrgica variará dependiendo del tipo de Fallot.

1. Fallot leve: tronco pulmonar normal, anillo pulmonar de diámetro normal, infundíbulo (hipertrofia difusa o estenosis infundibular limitada. Se realiza esternotomía media, seguida por extracorpórea convencional.

2. Fallot intermedio: Anillo de diámetro normal e infundíbulo hipoclásico, Anillo hipoplásico e infundíbulo con hipertrofia difusa. Se realiza ventriculotomía vertical.

3. Fallot severo: Tronco de diámetro hipoplasico Anillo hipoplásico. Infundíbulo hipoplásico. Se procede de forma similar a las descritas anteriormente, con la diferencia de que la incisión del tracto de salida se prolonga desde el infundíbulo a través del anillo y tronco pulmonar hasta un centímetro de la rama izquierda. 


\section{Manejo postoperatorio:}

Técnica paliativa:

1. Mantener un flujo adecuado en la fístula con una hidratacíón adecuada, manteniendo una buena tensión arterial y si es necesario administración de. Inotrópicos.

2. Mantener las resistencias vasculares periféricas bajas.

3. Garantizar el flujo, evitando presiones intratorácias altas, con P.I.P. bajos y altas frecuencias durante la ventilación mecánica y si es necesario con óxido nítrico.

4. Vigilar las Sat02 trascutáneas, deben mantenerse entre $75 \%$ y $85 \%$.

5. Auscultar la fistula en busca de un murmullo suave y constante, una vez retirada la ventilación mecánica.

Si el niño esta entubado y sometido a ventilación mecánica, se ausculta la fistula desconectado del ventilador y auscultando el tórax del niño o a través del T.E.T. ya que el extremo del tubo está situado al lado de la fistula

6. Antiagregar al niño, con ácido acetilsalisílico, para evitar la trombosis de la fistula.

7. Eliminar las burbujas de aire de los sistemas de infusión I.V. ya que hay shunt izquierda - derecha y pueden pasar a la circulación sistémica porque no tienen el filtro pulmonar

Corrección completa:

Los principales factores determinantes de graves complicaciones son el bajo gasto cardiaco, por lo que se mantendrá una presión venosa central alta después de la operación (aproximadamente entre 8-10) para mejorar la contractilidad y la utilización de inotrópicos convencionales como la dopamna, milrinona con efecto vasodilatador,

Alteraciones en la conducción:

La taquicardía ectópica de la unión, es una arritmia potencialmente seria. El ECG revela disociación AV con frecuencias altas (aproximadamente 180,200 1/m). La terapia utilizada es hipotermia moderada ( $33^{\circ}$-35 C.), disminuir la utilización de catecolaminas. Utilización de fármacos, digoxina, procainamida, amiodarona. Bloqueo de rama derecha (BRA) En casos de ventriculotomia derecha que en el postoperatorio inmediato se utiliza el marcapasos temporal, ya que en el momento de la intervención se colocan electrodos para la estimulación temporal. Si el bloqueo completo persiste en el tiempo se utilizará marcapasos permanente. Las arritmias ventriculares se reconocen como una complicación tardía de la reparación de la tretada de Fallot y se asocian con una alta frecuencia de muerte. súbita.

Disfunción ventricular derecha. El ventrículo derecho en la Tetralogía de Fallot, es pequeño y a menudo sufre complicaciones en el Postoperatorio, la acumulación de efectos como bypass, la incisión ventricular, la regurgitación pulmonar y quizás una comunicación interventricular residual o una obstrucción del tracto de salida del ventrículo derecho, dará 
lugar a un bajo gasto cardiaco con una elevada presión venosa central. El tratamiento consistirá en soporte ínotrópico y diuréticos, manteniendo una ventilación adecuada para disminuir las resistencias vasculares pulmonares Mantener el paciente hiperventilado con una PC02 por debajo de $40 \mathrm{~mm}$ de $\mathrm{Hg}$, utilización de vaso dilatadores como las prostaglandinas, la milrinona o el nitroprusiato y la administración de óxido nitríco como vasodilatador pulmonar selectivo a través de la vía respiratoria.

\section{Conclusiones}

- La tetralogía de Fallot es un trastorno cianótico congénito del corazón que abarca cuatro características anatómicas: hipertrofia ventricular derecha, defecto del tabique ventricular, anulación de la aorta y obstrucción del flujo de salida del ventrículo derecho. En los Estados Unidos, la prevalencia de Tetralogía deFallot es de aproximadamente 3.9 por 10,000 nacidos vivos, representando del 7 al 10 por ciento de las cardiopatías congénitas.

- Aunque suele aparecer esporádicamente sin otras anomalías, puede estar presente como parte de un síndrome conocido o trastorno genético, como el síndrome de Down. Otros asociados cardiacos. anomalías ocurren en aproximadamente el 40 por ciento de los pacientes con esta enfermedad.

- La presentación clínica depende del grado de obstrucción del flujo del retorno venoso y determina si hay una derivación de izquierda a derecha (acianótica) o de derecha a izquierda. En general, cuanto antes el inicio de la hipoxemia sistémica, más probable es que la estenosis del tracto de salida pulmonar sea grave o que la atresia esté presente. Además de la cianosis persistente en pacientes gravemente afectados, otros hallazgos incluyen episodios hipercianóticos (tet) intermitentes, crescendodecrescendo, soplo áspero de eyección sistólica, y segundo ruido cardiaco acentuado.

- Es importante la detección y tratamiento tempranos del paciente, debido a que su retraso conlleva a repercusiones importantes. La gran prevalencia de la enfermedad obliga a conocer su perfil y datos clave para su diagnóstico desde la evaluación temprana del infante con el objetivo de reducir al máximo posible la aparición de complicaciones futuras.

- Es importante la detección y tratamiento tempranos del paciente, debido a que su retraso conlleva a repercusiones importantes. La gran prevalencia de la enfermedad obliga a conocer su perfil y datos clave para su diagnóstico desde la evaluación temprana del infante con el objetivo de reducir al máximo posible la aparición de complicaciones futuras.

\section{Agradecimientos}

- Al Dr. Luis Barrezueta, Jefe de Docencia y al Dr. Simón Duque, Jefe de Servicio de Cardiología del Hospital de Niños "Dr. Roberto Gilbert Elizalde”. 


\section{Referencias bibliográficas.}

Doyle, T., \& Kavanaugh-McHugh, A. (Febrero de 2018).

Pathophysiology, clinical features, and diagnosis of Tetralogy of Fallot -. UpToDate, 1-11.

Mavroudis, C., \& Backer, C. (2013).

Pediatric cardiac surgery. Blackwell Publishing Ltd.

Nichols, D. G., Greeley, W. J., Lappe, D. G., Ungerleider, R. M., Cameron, D. E., Spevak, P. J., \& Wetzel, R. C. (2006).

Critical Heart Disease in Infants and Children E-Book. Elsevier Health Sciences.

Park, M. K. (2015).

Park Cardiologia Pediátrica. Elsevier Brasil.

Strong, W. B. (1992).

Nadas' Pediatric Cardiology. American Journal of Diseases of Children, 146(12), 1492-1493. 


\section{Para citar el artículo indexado.}

Moncayo A. \& Hernández M. (2019) Tetralogía de Fallot: diagnóstico, cirugía correctiva y manejo postoperatorio en Unidad de Cuidados Intensivos Cardiológicos Pediátricos, presentación de caso clínico y revisión bibliográfica. Revista electrónica Ciencia Digital 3(1), 35-53. Recuperado desde:

http://cienciadigital.org/revistacienciadigital2/index.php/CienciaDigital/article/view/249/55

$\underline{2}$

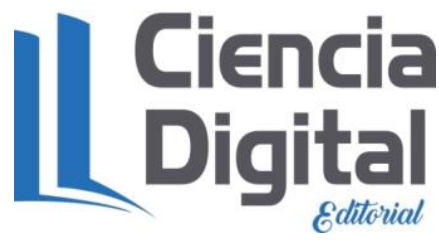

El artículo que se publica es de exclusiva responsabilidad de los autores y no necesariamente reflejan el pensamiento de la Revista Ciencia Digital.

El articulo queda en propiedad de la revista y, por tanto, su publicación parcial y/o total en otro medio tiene que ser autorizado por el director de la Revista Ciencia Digital.
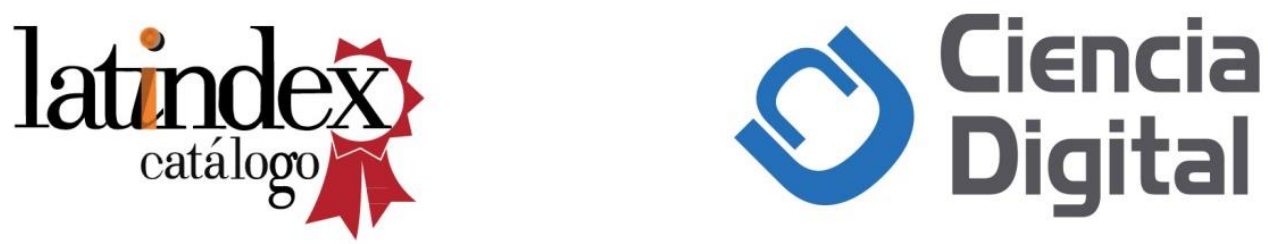